\title{
Álcool para controle de cascudinho em cama de frangos de corte
}

\author{
Fogaça, I. ${ }^{\text {; }}$ Ferreira, E. ${ }^{2}$; Saturnino, K.C. ${ }^{2}$; Santos, T.R. ${ }^{2}$; Cavali, J. ${ }^{2}$ e Porto, M.O. ${ }^{2}$
}

'Programa de Pós-Graduação em Ciências Ambientais. Universidade Federal de Rondônia. Rolim de Moura. RO. Brasil.

2Departamento de Medicina Veterinária. Universidade Federal de Rondônia. Rolim de Moura. RO. Brasil.

\author{
PaLAVRAS CHAVE-ADICIONAIS \\ Alphitobius diaperinus. \\ Ambiência. \\ Controle de praga.
}

\section{RESUMO}

O Brasil é o maior exportador e o terceiro maior produtor mundial de carne de frango, sendo a atividade geradora de grande quantidade de resíduo chamado de cama de frango. Este resíduo gera condições favoráveis para o desenvolvimento do besouro Alphitobius diaperinus (Panzer) (Coleoptera: Tenebrionidae), conhecido como cascudinho, sendo esse considerado a principal praga da avicultura, e afeta a produção avícola em todo o mundo. Seu controle é considerado difícil, sendo comumente empregadas substâncias piretróides e organofosforadas, apesar de não eficientes. Na busca pelo controle alternativo do inseto, vários métodos aditivos e inseticidas naturais vêm sendo testados. Contudo, na prática entomológica, substâncias com elevada pressão de vapor a fim de se produzir gases tóxicos são utilizadas para fixação dos insetos. Assim, em outubro de 2015, avaliou-se o álcool etílico PA para o controle de A. diaperinus em cama de frango. As unidades experimentais foram representadas por garrafas plásticas tipo pet cortadas, onde foram colocadas $100 \mathrm{~g}$ de cama de frango, acrescidos de 20 insetos adultos e 135 larvas, cada uma. Os tratamentos foram constituídos pela aplicação de álcool no volume de 1, 2,5, 5, 7,5 e $10 \mathrm{~mL}$ e sua diluição com água $(1: 1)$ sendo aplicado igual volume. Simularam-se dois ambientes, sendo um aberto (com telas contendo os insetos) e um fechado (recipiente amostral fechado com a porção previamente cortada da garrafa petf. Nas parcelas foram mantidas a temperatura de $28,5 \pm 2{ }^{\circ} \mathrm{C}$ e UR de $51,0 \pm 2 \%$, feito seis repetições por tratamento. A quantidade de $2,5 \mathrm{~mL}(2,5 \% \mathrm{v} / \mathrm{p})$ de álcool gerou $100 \%$ de mortalidade de adultos e larvas nas amostras fechadas, sendo necessário o dobro do volume no sistema aberto. Para a diluição álcool-água (1:1) o controle de 100\% foi obtido com o dobro das quantidades relatadas anteriormente em relação aos respectivos tratamentos.

\section{Alcohol to control dark beetle in poultry litter}

\section{SUMMARY}

\section{ADDITIONAL KEYWORDS}

Alphitobius diaperinus.

Ambience.

Pest control.

\section{INFORMATION}

\section{Cronología del artículo.}

Recibido/Received: 26.02.2016

Aceptado/Accepted: 25.05.2017

On-line: 15.10 .2017

Correspondencia a los autores/Contact e-mail:

elvino@unir.br

\section{INTRODUÇÃO}

Alphitobius diaperinus (Panzer) pode ser considerado o principal inseto praga veiculado a cama de frangos de corte. Originário da África Ocidental foi introduzido em outros países por meio de alimentos contaminados e, com o sistema de criação intensivo, achou condições propícias para seu desenvolvimento no ambiente avícola (Salin et al., 2000).

Brazil is the third largest producer of chicken meat in the world, resulting in the production of a large amount of waste called chicken litter. This residue generates favorable conditions for the development of the beetle Alphitobius diaperinus (Panzer) (Coleoptera: Tenebrionidae), known as cascudinho, which is considered the main pest of poultry farming, affecting poultry production worldwide. Its control is considered difficult, and pyrethroid and organophosphorous substances are commonly used, although they are not efficient. In the search for alternative insect control, several natural insecticidal and insecticidal methods have been tested. However, in the entomological practice, substances with high vapor pressure in order to produce toxic gases are used for insect fixation. Thus, in October 2015, ethyl alcohol PA was tested with the aim of controlling $A$. diaperinus in chicken litter. The experimental units were represented by cut plastic pet bottles, where $100 \mathrm{~g}$ of were poultry litter added, with 20 adult insects and 135 larvae each. The treatments were constituted by the application of alcohol in the volume of 1, 2.5, 5, 7.5 and $10 \mathrm{~mL}$ and its with dilution water $(1: 1)$ with equal volume applied. Two environments were simulated, one open (screens to contain the insects) and one closed (sample container closed with the portion previously cut). The plots were maintained at $28.5 \pm 2{ }^{\circ} \mathrm{C}$ and $51.0 \pm 2 \%$ of relative humidity, with six replicates per treatment. The amount of $2.5 \mathrm{~mL}(2.5 \% \mathrm{v} / \mathrm{p})$ alcohol generated $100 \%$ mortality in adults and larvae in the closed samples, requiring twice the volume in the open system. For the alcohol-water dilution $(1: 1)$ the $100 \%$ control was obtained with twice the amount reported previously in relation to the respective treatments. 
Coronavirus, bactérias (p.ex. Micrococcus sp., Streptococcus sp., Corynebacterium sp., Bacillus subtilis, Staphylococcus aureus, Proteus mirabilis, P. vulgaris, Paracolobactrum intermedium, Escherichia coli, E. intermedia, E. freundii, Serratia marcescens, Klebsiella-Aerobacter, Pseudomonas aeruginosa e Salmonella sp.), fungos (p.ex. Aspergillusflavus, A. niger, A. repens, A. candidus, Penicillium sp. e Candida sp.) e protozoários como Eimeria sp. (ChernakiLeffer et al., 2002; Vitori et al., 2007).

Dentre as bactérias, as do gênero Salmonella podem ser destacadas devido sua importância na avicultura e ao risco de contaminação alimentar em seres humanos. Essa bactéria já foi isolada de $A$. diaperinus e sua presença é rotineiramente verificada na cama dos aviários. Também E. coli é bastante frequente em granjas avícolas, tanto na cama, como no inseto, que contribui em sua disseminação (Chernaki-Leffer et al., 2002).

O conhecimento da flutuação populacional e da distribuição espacial dessa praga é de fundamental importância para o estabelecimento de uma metodologia de controle adequada. Sabe-se que a distribuição da estrutura populacional desse inseto no solo de galpões de avicultura é altamente heterogênea havendo maior quantidade de larvas, pupas e adultos sob os comedouros (Salin et al., 2000; Uemura et al., 2008). Seu ciclo biológico é inconstante e depende das condições de temperatura, umidade e disponibilidade de alimento (Paiva, 2000), contudo, o ambiente criatório de aves de corte gera condições em que não se observa relação entre as variações de temperatura do galpão e da cama e o número de insetos coletados semanalmente (Chernaki-Leffer et al., 2007).

$\mathrm{O}$ ciclo completo de $A$. diaperinus à temperatura constante de $28^{\circ} \mathrm{C}$ é de 42,5 dias, o que indica que a cada lote introduzido na granja (o que ocorre a cada 50 dias, aproximadamente) pode ocorrer uma nova geração de insetos. Cada fêmea pode produzir acima de 2 mil ovos (Steelman, 1996). Em temperatura de $27^{\circ} \mathrm{C}$ e umidade relativa do ar (UR) de $80 \%$, a eclosão das larvas ocorre em 3 a 10 dias, as quais sofrem de 6 a 10 mudas no período de 15 a 20 dias, atingindo o estádio de pupa. Estas se desenvolvem de 4 a 14 dias, quando se tornam adultas e podem viver por mais de um ano. As fêmeas iniciam a postura em 6 a 10 dias após o acasalamento (Paiva, 2000). Em relação a sua adaptação registra-se que sob regime de flutuação térmica em baixas temperaturas, esses insetos podem reparar injúrias quando a temperatura de conforto térmico é estabelecida (Colinet et al., 2011).

Para as estratégias de controle, normalmente inseticidas industriais são utilizados, mas devido a presença das aves nos galpões há dificuldade em seu emprego uma vez que podem causar intoxicação nos animais. Assim sua aplicação se dá em condições de vazio sanitário. Contudo a prevenção ou o controle do inseto por esse meio pode gerar problemas. Sabe-se que a avicultura tem sido considerada como atividade de grande impacto pelos órgãos de controle ambiental, devido ao seu potencial poluidor, pois produz grande quantidade de cama, carcaças de animais mortos e águas residuárias (Silva, 2005). Tal situação ocorre pelo confinamento e a intensificação da produção que traz como consequência o aumento do volume de dejetos produzidos por unidade de área. Esses resíduos continuam a serem lançados em cursos d'agua, estocados e ou descarregados a céu aberto, sem qualquer tratamento, transformando-se em fonte poluidora e constituindo fator de risco para a saúde humana e animal (Matos et al., 1998). Contudo, tem-se com as práticas de entomologia o uso de substâncias que produzem gases tóxicos aos insetos a fim de se fazer sua coleção e estudo. Dentre eles acetado de etila, éter e clorofórmio podem ser citados (Gallo et al., 1978). Como o álcool é uma substância também usada e de baixa toxicidade ao ser humano, quando utilizada de maneira correta, aventou-se a hipótese de que seu emprego poderia controlar a população de cascudinhos.

Cabe ressaltar que o aproveitamento da cama de frangos como fornecedor de nutrientes para os vegetais atende tanto os conceitos do agronegócio como os das leis de proteção ambiental e sanitária. Como desde 2001 o emprego de cama de frango e de qualquer outro produto de origem animal está proibido para alimentação de ruminantes (Brasil, 2004), tem se a necessidade de estudo de sua viabilidade como fontes de nutrientes, criando uma alternativa para seu uso. É importante lembrar que a criação de frangos produz cerca de quatro toneladas de cama a cada 1000 aves abatidas (Konzen, 2003) e atualmente há preocupação ambiental relativa ao potencial contaminante quando do emprego de camas tratadas por muitos ciclos com inseticidas industriais são empregadas em pastagens ou em hortaliças.

Sob estes contexto, o presente estudo objetivou avaliar a eficácia do uso de álcool etílico PA em diferentes concentrações para o controle de $A$. diaperinus em cama de frango.

\section{MATERIAL E MÉTODOS}

O estudo teve início em 13 de junho de 2015, na granja Sonai, linha $188 \mathrm{~km}$ 03-Sul, no município de Rolim de Moura, Rondônia (latitude $11^{\circ} 48^{\prime} 13^{\prime \prime}$ Sul e longitude $61^{\circ} 48^{\prime} 12^{\prime \prime}$ Oeste, a $277 \mathrm{~m}$ de altitude, $\mathrm{T} \sim 24$ - $32^{\circ} \mathrm{C}$, UR $\sim 85 \%$ e índice pluviométrico entre 2000 e $2500 \mathrm{~mm}$ ). O clima da região é o Aw da classificação de Köppen-Geiger (Pell et al., 2007), caracterizado como equatorial com variação para o tropical quente e úmido e estação seca bem definida (março a setembro).

O local de estudo possui dois galpões (14,5x126 m e $15 \times 127 \mathrm{~m}$ ) com capacidade de alojamento inicial de 51 mil aves, utilizando cama de frangos a base de maravalha. Seu manejo constitui-se na adição de nova camada de pequena espessura após revolvimento com microtrator motocultivador de solo a combustão no período de vazio sanitário, de duração de 5 a 7 dias. Nesta mesma etapa, ocorre aspersão de inseticida a base de Cipermetrina $6 \%$, na dose de $3,8 \mathrm{~g} \mathrm{~m}^{-2}$, conforme recomendação do fabricante (entre 3 a $5 \mathrm{~g} \mathrm{~m}^{-2}$ ).

No intuito de estudar o efeito do álcool, insetos (adultos e larvas de diversos estágios) foram coletados dos galpões, aleatoriamente, e levados ao Laboratório Multidisciplinar da Universidade Federal de Rondônia/Campus Rolim de Moura. Como umidades experimentais foram usadas garrafas plásticas tipo pet. Estas foram cortadas a $15 \mathrm{~cm}$ de sua base de forma a permitir o encaixe de sua parte superior. Nestes recipientes foram colocadas $100 \mathrm{~g}$ de cama de frango, acrescidos 
de 20 insetos adultos e 135 larvas em diversos estágios de desenvolvimento, cada uma. Os tratamentos foram constituídos pela aplicação de álcool etílico PA nos volumes de 1, 2,5, 5, 7,5 e $10 \mathrm{~mL}$ e sua diluição com água (1:1) com igual volume aplicado. Para se simular diferentes ambientes, procedeu-se a contenção dos insetos com o uso de tela $(\varnothing 1 \mathrm{~mm})$ preza na abertura das garrafas com elástico (ambiente aberto a trocas gasosas) e outro, onde os recipientes eram tampados com a parte superior da garrafa cortada (ambiente fechado, dificultando as trocas gasosas). As parcelas foram mantidas a temperatura de $28,5 \pm 2{ }^{\circ} \mathrm{C}$ e UR de $51,0 \pm$ $2 \%$, havendo seis repetições por tratamento. Contouse também com um tratamento testemunha absoluta e outro com a aplicação de $10 \mathrm{~mL}$ de água.

O álcool e as soluções foram aplicados com o uso de pipeta graduada no meio da massa de cama. Vinte e quatro horas após a aplicação os insetos mortos foram contados, assim como após 48 horas da aplicação. $\mathrm{O}$ inseto adulto foi considerado morto quanto se apresentava em decúbito dorsal sem movimentação das patas. Larvas quando não apresentavam movimentação corporal (Gazoni et al., 2012).

Para comparação entre os tratamentos, utilizou-se o delineamento inteiramente casualizado sendo o contraste de médias feito através do teste de Scott-Knot ao nível de $5 \%$ de probabilidade. Todas as análises foram realizadas com auxílio do software livre $\mathrm{R}$ (Pinheiro et al., 2014), sendo os dados obtidos após 24 horas da aplicação dos tratamentos, por não diferir da contagem com 48 horas.

\section{RESULTADOS E DISCUSSÃO}

Atualmente para o controle de pragas, além do aspecto de dano econômico, há a preocupação quanto ao potencial contaminante ao ambiente relativo aos produtos utilizados para controle dos insetos (OviedoRondón, 2008; Wolf et al., 2014). Neste estudo, onde foram avaliados os tratamentos contendo álcool etílico PA e sua diluição em água (1:1) pôde ser observado, para alguns tratamentos que, após 20 minutos da aplicação ocorreu mortalidade dos insetos adultos.

O quantitativo entre mortos e vivos não apresentou mudança com 24 e 48 horas. Com a aplicação de $1 \mathrm{~mL}$ de álcool não se obteve efeitos importantes quanto a mortalidade dos adultos. Contudo, quando aplicados 2,5 mL de álcool em 100g de cama de frangos, obtevese $100 \%$ de mortalidade de adultos em condições de maior dificuldade entre as trocas gasosas com o meio externo (ambiente fechado). Quando não houve tal impedimento, ou seja, a retenção dos insetos era feita com o uso de tela, tal nível de mortalidade foi obtido com o dobro da quantidade de álcool aplicada (tabela I). Os mesmos resultados (100\% mortalidade) foram também obtidos com as maiores doses.

Para as estratégias de controle, normalmente inseticidas industriais são utilizados, mas devido a presença das aves nos galpões há dificuldade em seu emprego uma vez que podem causar intoxicação nos animais. Assim sua aplicação se dá em condições de vazio sanitário. Contudo a prevenção ou o controle do inseto por esse meio pode gerar outros problemas como o uso da cama de frangos, como visto no uso de organoarsenicais que, quando empregados como fertilizante

\begin{tabular}{|c|c|c|c|c|}
\hline \multirow[t]{2}{*}{ Tratamentos } & \multicolumn{2}{|c|}{ Numero de adultos mortos em ambiente } & \multicolumn{2}{|c|}{$\%$ Mortalidade } \\
\hline & Fechado $^{1}$ & Aberto $^{2}$ & Fechado & Aberto \\
\hline Testemunha absoluta & $0,0^{\mathrm{d}}$ & $0,0^{c}$ & 0 & 0 \\
\hline Testemunha $10 \mathrm{~mL}$ água & $0,0^{\mathrm{d}}$ & $0,0^{c}$ & 0 & 0 \\
\hline $1 \mathrm{~mL}$ álcool & $4,3^{c}$ & $0,0^{c}$ & 22 & 0 \\
\hline $2,5 \mathrm{~mL}$ álcool & $20,0^{\mathrm{a}}$ & $0,0^{c}$ & 100 & 0 \\
\hline $5,0 \mathrm{~mL}$ álcool & $20,0^{\mathrm{a}}$ & $20,0^{\mathrm{a}}$ & 100 & 100 \\
\hline 7,5 mL álcool & $20,0^{\mathrm{a}}$ & $20,0^{\mathrm{a}}$ & 100 & 100 \\
\hline 10 mL álcool & $20,0^{\mathrm{a}}$ & $20,0^{\mathrm{a}}$ & 100 & 100 \\
\hline 1 mL água-álcool (1:1) & $0,0^{\mathrm{d}}$ & $0,0^{c}$ & 0 & 0 \\
\hline 2,5 mL água-álcool (1:1) & $11,2^{\mathrm{b}}$ & $0,0^{c}$ & 56 & 0 \\
\hline 5,0 mL água-álcool (1:1) & $20,0^{\mathrm{a}}$ & $0,0^{c}$ & 100 & 0 \\
\hline 7,5 mL água-álcool (1:1) & $20,0^{\mathrm{a}}$ & $3,58^{b}$ & 100 & 18 \\
\hline 10 mL água-álcool (1:1) & $20,0^{\mathrm{a}}$ & $20,0^{a}$ & 100 & 100 \\
\hline
\end{tabular}

${ }^{1}$ Ambiente fechado: recipientes tampados com a parte superior da garrafa cortada. ${ }^{2} \mathrm{Ambiente}$ aberto: uso de tela (1 mm) presa com elástico na abertura das garrafas plásticas. Médias seguidas pela mesma letra nas colunas não diferem estatisticamente pelo teste de Scott-Knot a $5 \%$ de probabilidade. 
nos agroecossistemas, são transformados em ácido dimetilarsônico (Dimethylarsonic acid - DMA), ácido monometilarsônico (monomethylarsonic acid- MMA) e arsênio inorgânico. Estes são mais tóxicos e móveis que os organoarsenicais e com grande potencial de contaminação de águas. Por este motivo foram banidos da Europa deste 1998 e do estado Americano de Maryland-USA deste 2012 (Mangalgiri et al., 2015). No Brasil o controle de $A$ diaperinus se baseia na aplicação de cipermetrina, derivado da piretrina e piretróides $\left(\mathrm{C}_{22} \mathrm{H}_{19} \mathrm{Cl}_{2} \mathrm{NO}_{3}\right.$ - Classe toxicológica II) que, apesar de fotoestável, biodegradável e com baixa toxicidade para animais homeotérmicos, é tóxico para peixes (Montana et al., 2012; Montana et al., 2014) e considerado ineficiente para condições de piso cimentado ou de terra batida (Uemura et al., 2008).

Outra maneira de controle se baseia na utilização de agentes biológicos que geram menor impacto ao ambiente. Nesta proposição relatam-se a suscetibilidade do cascudinho a isolados de nematóides entomopatogênicos como Steinernema glaseri, S. feltiae e Heterorhabditis heliothidis (Nematoda: Rhabtida), tanto para larvas como para adultos. Dentre os isolados avaliados, destacaram-se S. carpocapsae - ARO e S. carpocapsae UFEND que proporcionaram valores médios de mortalidade confirmada de $48 \%$ e $40 \%$, respectivamente. Por outro lado, S. glaseri demonstrou ser pouco patogênico, visto que o maior percentual de mortalidade foi de 3,3\%, obtido na maior concentração. Também há estudo que avaliou a eficiência de Beauveria bassiana, terra diatomácea e microrganismos eficazes (EM-4: composto por bactérias ácido-láticas - Lactobacillus e Pediococcus, leveduras - Sacharomyces, bactérias fotossintéticas e actinomicetos - todos compatíveis entre si), e sua associação, no controle de Alphitobius diaperinus. Os maiores índices de mortalidade foram observados nas associações de Terra de diatomácea com Beauveria bassiana e estes com EM-4. O uso conjunto de B. bassiana e terra diatomácea podem reduzir o uso de produtos químicos, para o manejo de controle das populações de A. diaperinus (Santoro et al., 2008).

Outra proposta de estudo para controle do cascudinho está no uso de Nim-indiano (Azadirachta indica A. Juss. - Meliaceae) uma vez que seu óleo promove altos níveis de proteção (até 150 dias) contra Zabrotes subfasciatus (Boheman) (Coleoptera: Bruchidae) - caruncho do feijão armazenado. No tratamento de sementes, proporciona a mesma proteção quanto ao uso de malathion (Barbosa et al., 2002).

Com esse estudo nos tratamentos de diluição, os efeitos de mortalidade de adultos (56\%) foram observados a partir da aplicação de $2,5 \mathrm{~mL}$ (ambiente fechado) e $7,5 \mathrm{~mL}$ (18\%; ambiente aberto), também sendo observados níveis de mortalidade de $100 \%$ a partir das maiores doses anteriormente relatadas (tabela I).

Resultados semelhantes foram observados com as larvas sendo a mortalidade obtida em volumes menores de álcool e sua diluição, em relação aos adultos (tabela II). Certamente, a elevada pressão de vapor do álcool está relacionada com esses resultados, por promover um colapso traqueal e comprometer o acesso do oxigênio ao sistema respiratório do inseto. Contudo o uso de álcool pode representar um risco quanto a incêndios nas instalações e sua solução aquosa pode ser uma maneira de eliminar tal risco. Deve ser observado, todavia, que o nível de umidade aportado a cama não deve ser elevado já que sua função é a de promover a

\begin{tabular}{|c|c|c|c|c|}
\hline \multirow{2}{*}{ Tratamentos } & \multicolumn{2}{|c|}{ Numero de larvas mortas em ambientes } & \multicolumn{2}{|c|}{ Mortalidade (\%) } \\
\hline & Fechado $^{1}$ & Aberto $^{2}$ & Fechado & Aberto \\
\hline Testemunha absoluta & $0,0^{c}$ & $0,0^{\mathrm{d}}$ & 0 & 0 \\
\hline Testemunha $10 \mathrm{~mL}$ água & $0,0^{c}$ & $0,0^{d}$ & 0 & 0 \\
\hline 1 mL álcool & $135,0^{\mathrm{a}}$ & $0,0^{d}$ & 100 & 0 \\
\hline 2,5 mL álcool & $135,0^{\mathrm{a}}$ & $54,0^{\circ}$ & 100 & 40 \\
\hline 5,0 mL álcool & $135,0^{\mathrm{a}}$ & $135,0^{\mathrm{a}}$ & 100 & 100 \\
\hline 7,5 mL álcool & $135,0^{\mathrm{a}}$ & $135,0^{\mathrm{a}}$ & 100 & 100 \\
\hline $10 \mathrm{~mL}$ álcool & $135,0^{\mathrm{a}}$ & $135,0^{\mathrm{a}}$ & 100 & 100 \\
\hline 1 mL água-álcool (1:1) & $27,0^{\mathrm{b}}$ & $0,0^{d}$ & 20 & 0 \\
\hline 2,5 mL água-álcool (1:1) & $135,0^{\mathrm{a}}$ & $0,0^{\mathrm{d}}$ & 100 & 0 \\
\hline 5,0 mL água-álcool (1:1) & $135,0^{\mathrm{a}}$ & $108,0^{\mathrm{b}}$ & 100 & 20 \\
\hline 7,5 mL água-álcool (1:1) & $135,0^{\mathrm{a}}$ & $135,0^{a}$ & 100 & 100 \\
\hline 10 mL água-álcool (1:1) & $135,0^{a}$ & $135,0^{\mathrm{a}}$ & 100 & 100 \\
\hline
\end{tabular}

${ }^{1}$ Ambiente fechado: recipientes tampados com a parte superior da garrafa cortada. ${ }^{2}$ Ambiente aberto: uso de tela ( $\left.1 \mathrm{~mm}\right)$ preza com elástico na abertura das garrafas plásticas. Médias seguidas pela mesma letra nas colunas não diferem estatisticamente pelo teste de Scott-Knot a $5 \%$ de probabilidade. 
retirada de umidade das fezes das aves (Avila et al., 1992; Oliveira e Carvalho, 2002).

Pode ser considerado que, mesmo quando pequeno, o controle de larva é fundamental, uma vez que essa é a fração de maior número do extrato populacional em condições de galpão por refletir a contínua atividade reprodutiva do inseto (Uemura et al., 2008). A fêmea pode produzir posturas com 2 mil ovos (Steelman, 1996) ou mesmo chegar a 200 ou 400 mil ovos em cada uma das 12 posturas anuais em condições de aviário (Pinto Júnior et al., 2010).

A maior ou menor exposição (ambiente fechado ou aberto) proporcionam diferentes níveis de resposta quanto à mortalidade de adultos e larvas. Como se obteve resposta tanto para o uso de álcool como em sua diluição, tratamentos com álcool gel poderiam ser propostos para gerar condições de aplicação pontual no galpão devido a heterogeneidade da distribuição populacional do inseto (Salin et al., 2000; Uemura et al., 2008) e mesmo na condição de vazio sanitário, já que instalações com piso de terra geram melhores condições para o inseto empupar do que em piso de cimento, facilitando a reinfestação (Uemura et al., 2008).

Existe grande interesse em comprometer o desenvolvimento normal da população de cascudinhos até que seja possível a aplicação de produto diretamente no solo, na oportunidade de substituição da cama do galpão e tornar o controle do inseto mais efetivo. $\mathrm{O}$ manejo tradicional em avicultura industrial envolve a aplicação de inseticidas industriais em todo aviário no momento do vazio sanitário e, com a troca da cama, há a lavagem e desinfecção das instalações. Nesta oportunidade não pode ser descartado o risco da contaminação de corpos d'água e, até mesmo a intoxicação de peixes, já que no Brasil o controle de A. diaperinus é baseado na aplicação de cipermetrina (Montana et al., 2012; Montana et al., 2014).

O uso de cipermetrina, clorpiriflos e citronelal (5 L $\mathrm{m}^{-2}$ de calda) no solo dos galpões gera eficiência na redução de larvas e adultos devido os insetos serem atingidos com o encharcamento do solo. A avaliação com cipermetrina ( $1 \%$ ou $1 \mathrm{~L} / 100 \mathrm{~L})$ com volume de calda de 20 , 40 e $80 \mathrm{~L} \mathrm{~m}^{-2}$ reduziram em mais de $95 \%$ a emergência dos insetos adultos. A infiltração desuniforme, em função de diferentes níveis de compactação deve ser considerada, contudo, o hábito de cavar galerias para empupar (até $10 \mathrm{~cm}$ ) permite maior infiltração do princípio ativo e controle do inseto (Santos et al., 2009). Para a aplicação de álcool ou solução aquo-alcoólica o nível de perda de álcool por evaporação deve ser avaliado.

Inseticidas naturais também são interessantes como estratégia de controle sendo relatada maior susceptibilidade de adultos e larvas de cascudinho para o óleo de sassafrás (Ocotea odorífera; $\mathrm{CL}_{50}$ adulto: 0,26 $\mathrm{mL} \mathrm{L}^{-1} \mathrm{e}$ $\mathrm{CL}_{50}$ larvas: $0,12 \mathrm{~mL} \mathrm{~L}^{-1}$ ) do que para óleo de eucalipto (Eucapyptus viminalis; $\mathrm{CL}_{50}$ adulto: $0,37 \mathrm{~mL} \mathrm{~L}^{-1}$ e $\mathrm{CL}_{50}$ larvas: 1,72 $\mathrm{mL} \mathrm{L}^{-1}$ ) (Pinto Júnior et al., 2010).

O Nim indiano (Azadirachta indica) apresenta atividade inseticida contra cerca de 400 espécies de pragas devido à presença de azadiractina, um tetranortriterpenóide de baixíssima toxicidade para animais domésticos e para o homem. O uso de sua torta $\left(100 \mathrm{~g} \mathrm{~m}^{-2}\right) \mathrm{em}$ cama de frango não afetou a sobrevivência dos insetos, mas reduziu a ovoposição (23\%) e a atividade alimen$\operatorname{tar}(21 \%)$ (Alves et al., 2012).

Neste estudo foi obtido nível de mortalidade máximo com o uso de álcool como princípio ativo. Em um cenário ideal a ausência de Alphitobius diaperinus em galpões de avicultura industrial é desejada, já que esse inseto é ingerido, preferencialmente do $4^{\circ}$ ao $20^{\circ}$ dia de vida da ave, o que atrapalha seu balanço nutricional e desempenho (Uemura et al., 2008; Pinto Júnior et al., 2010). No englúvio (papo) e moela os insetos ingeridos provocam perfurações que podem levar o animal a óbito. Adultos e larvas perfuram a pele das aves para se alimentar de exsudato sanguíneo. Além disso, sua presença no trato intestinal da ave representa risco de contaminação da carcaça quando em seu processamento. Neste caso, com a extração do papo e da moela, há potencial risco de transmissão de patógenos bacterianos, virais, fúngicos, de protozoários e platelmintos, inclusive coronavírus (Uemura et al., 2008).

Na literatura, a eliminação total do Alphitobius diaperinus é obtida com a aplicação de fosfina, gás extremamente tóxico, que atua inibindo parcialmente a cadeia de transporte de elétrons com aumento da produção de superóxido dismutase e radical hidroxil, o que acarreta a peroxidação de lipídeos da membrana celular comprometendo a célula e levando o inseto a morte (Gazoni et al., 2012). Níveis de 100\% de mortalidade foram conseguidos com esse estudo com o uso de álcool e solução aquo-alcoólica representando uma fonte não poluidora e sem efeito residual para o ambiente do galpão. Estudos com esse intuito são desenvolvidos com o uso da temperatura a fim de se obterem métodos alternativos que não gerem riscos para a sanidade das aves e para saúde humana.

Relata-se que temperaturas inferiores a $-10^{\circ} \mathrm{C}$ ou superiores a $45^{\circ} \mathrm{C}$ promovem nível máximo de mortalidade para Alphitobius diaperinus avaliados em condições de laboratório (em placas de Petri). As baixas temperaturas podem representar uma tecnologia para países ou regiões de clima temperado na situação de inverno. Para temperaturas elevadas pode se contar com o uso de lona e da umidade da cama no final do ciclo para desencadear o processo de fermentação (Gazoni et al., 2012), contudo tal metodologia tem aplicação no período de vazio sanitário que normalmente é de sete dias, mas que pode ser encurtado devido a demanda do integrador e com isso não proporcionar tempo suficiente para que o processo fermentativo atinja os níveis de temperatura desejada. Também deve ser considerado o custo de aquisição de lona e a mão de obra envolvida no procedimento e o tempo de desidratação da cama para que se tenha níveis de umidade baixos e satisfatórios a deposição das excretas das aves.

Cabe ressaltar que os insetos podem desenvolver resistência ao ingrediente ativo repetidamente utilizado em seu controle, como é o caso para cipermetrina, dichorvos e triflumuron (Chernaki-Leffer, 2004; Chernaki-Leffer et al., 2011). Muitas vezes os tratamentos com moléculas industriais se tornam ineficazes devido à alcalinidade presente na cama o que desestabiliza o princípio ativo do produto (cipermetrina e diclorvós, por exemplo) reduzindo o efeito residual e consequentemente causando a reinfestação por insetos resistentes (Santos et al., 2009). Essa prática, considerada ineficiente, além da seleção de insetos resistentes, geram 
intoxicação e eliminação de inimigos naturais (Alves et al., 2012).

Em um cenário ideal, ou seja, em que o emprego de fontes ou pastilhas alcoólicas promovesse $100 \%$ de mortalidade do inseto, não se teria efeito residual com também se eliminaria o risco da geração de população resistente. Contudo, caso isso não possa ser alcançado, o desenvolvimento de estudos visando o efeito inseticida por manejo integrado com outras substancias inseticidas naturais é de interesse no manejo de aves em agroecossistemas devido à possibilidade da ocorrência de indivíduos resistentes ao álcool como identificados para Drosophila melanogaster (Herrewege e David, 1980).

\section{CONCLUSÕES}

O uso de álcool ou de sua solução aquosa promoveu controle para Alphitobius diaperinus, verificando-se $100 \%$ de mortalidade para adultos e larvas com aplicação de 2,5 mL de álcool etílico PA para cada $100 \mathrm{~g}$ de cama, em ambiente com troca gasosa restringida em relação ao meio exterior. Quando a contenção dos insetos se deu somente por tela, necessitou-se de 5,0 mL para ser obtido o mesmo nível de mortalidade. Para o álcool diluído em água (1:1) o nível de mortalidade de $100 \%$ foi obtido com dobro dos volumes em relação aos tratamentos estudados.

\section{BIBLIOGRAFIA}

Alves, V.M.; Alves, F.A. e Uemura-Lima, D.H. 2012. Atividade da torta de nim sobre adultos do cascudinho dos aviários em condições de laboratório. Cienc Rural, 42: 888-893.

Avila, V.S.; Mazzuco, H. e Figueredo, S. 1992. Cama de aviário: materiais, reutilização, uso como alimento e fertilizante. Concórdia. EMBRAPA. Circular Técnica, 16: 38.

Barbosa, F.R.; Yokoyama, M.; Pereira, P.A.A. e Zimmermann, F.J.P. 2002. Controledocaruncho-do-feijoeiro Zabrotes subfasciatuscomóleos vegetais, munha, materiais inertes emalathion. Pesq Agropec Bras, 37: 1213-1217.

Brasil. 2004. Instrução Normativa 8 de 25 de março de 2004. Diário Oficial da União: 1,5

Chernaki-Leffer, A.M.; Biesdorf, S.M.; Almeida, L.M.; Leffer, E.V.B. e Vigne, F. 2002. Isolamento de enterobactérias em Alphitobius diaperinus e na cama de aviários no oeste do estado de Paraná, Brasil. Rev Bras Cienc Avic, 4: 243-347.

Chernaki-Leffer, A.M. 2004. Dinâmica populacional, estimativa da resistência a inseticidas e alternativas de controle para o cascudinho Alphitobius diaperinus (Panzer, 1797) (Coleoptera: Tenebrionidae). Tese (Doutorado em Ciências). Departamento de Ciências Biológicas. Universidade Federal do Paraná, Curitiba. 123 pp.

Chernaki-Leffer, A.M.; Almeida, L.M.; Sosa-Gómez, D.R.; Anjos, A. and Vogado, K.M. 2007. Populational fluctuation and spatial distribution of Alphitobius diaperinus (Panzer) (Coleoptera; Tenebrionidae) in a poultry house, Cascavel, Parana state, Brazil. Braz J Biol, 67: 209-213.

Chernaki-Leffer A.M.; Sosa-Gómez, D.R.; Almeida, L.M. and Lopes, I.O.N. 2011. Susceptibility of Alphitobius diaperinus (Panzer) (Coleoptera, Tenebrionidae) to cypermethrin, dichlorvos and triflumuron in southern Brazil. Rev Bras Entomol, 55: 125-128.

Colinet, H.; Lalouette, L. and Renault, D. 2011 . A model for the time-temperature-mortality relationship in the chill-susceptible beetle, Alphitobius diaperinus, exposed to fluctuating thermal regimes. J Therm Biol, 36: 403-408.

Gallo, D.; Nakano, O.; Silveira Neto, S.; Carvalho, R.P.L.; Batista, G.C.; Berti Filho, E.; Parra, J.R.P.; Zucchi, R.A. e Alves, S.B. 1978. Manual de Entomologia Agrícola. Ed. Agronômica Ceres. São Paulo. 531 pp.

Gazoni, F.L.; Flores, F.; Bampi, R.A.; Silveira, F.; Boufleur, R. e Lovato, M. 2012. Avaliação da resistência do cascudinho (Alphitobius diaperinus)
(Panzer) (Coleoptera: Tenebrionidae) a diferentes temperaturas. Arq Inst Biol, 79: 69-74

Herrewege, J. and David, J.R. 1980. Alcohol tolerance and alcohol utilization in Drosophila: Partial independence of two adaptive traits. Heredity, 44: 229-235.

Konzen, E. 2003. Fertilização da lavoura e pastagem com dejetos de suínos e cama de aves. IVSeminário Técnico da Cultura do Milho. Videira, SC. Brasil.

Matos, A.T.; Vidigal, S.M.; Sediyama, M.A.N.; Garcia, N.C.P. e Ribeiro, M.F. 1998. Compostagem de alguns resíduos orgâmicos, utilizando-se águas residuárias da suinocultura como fonte de nitrogênio. Rev Bras Eng Agríc Ambient 2: 199-203.

Mangalgiri, K.P.; Adak, A. and Blaney, L. 2015. Organoarsenicals in poultry litter. Detection, fate and toxicity. Environ International, 75: 68-80.

Montanha, F.P.; Galeb, L.A.G.; Mikos, J.D.; Ganeco, L.N.; Pereira, T P.; Tanaka, A.; Kirschnik, P.G. and Pimpão, C.T. 2012. Pyrethroid toxicity in silver catfish, Rhamdia quelen. Pesq Vet Bras, 32: 1297-1303.

Montanha, F.P.; Fredianelli, A.C.; Wagner, R.; Sacco, S.R.; Rocha, D.C.C. and Pimpão, C.T. 2014. Clinical, biochemical and haemathological effects in Rhamdia quelen exposed to cypermethrin. Arq Bras Med Vet Zootec, 66: 697-704.

Oliveira, M.C. e Carvalho, I.D. 2002. Rendimento e lesões em carcaças de frangos de corte criados em diferentes camas e densidades populacionais. Ciênc Agrotéc, 26: 1076-1081.

Oviedo-Rondón, E.O. 2008. Technologies to mitigate theenvironmental impact of broiler production. Rev Bras Zooten, 37 (Num. Special): 239-252.

Paiva, D.P. 2000. Controle de moscas e cascudinhos. Desafios na produção agrícola. Proceedings do Simpósio sobre resíduos da Produção Avícola. 12 Abril. Concórdia, SC. Brasil. pp. 21-26.

Peel,M.C.; Finlayson, B.L. and McMahon, T.A. 2007. Updated worldmap of the Köppen-Geiger climate classification. Hydrol Earth SystSc, 11: 1633-1644.

Pinheiro J.; Bates D.; DebRoy, S. and Sarkar, D. 2014. R Core Team.nlme: Linear and nonlinear mixed effects models. R package version 3. $117 \mathrm{pp}$. Pinto Junior, A.R.; Carvalho, R.I.N.;Netto, S.P.;Weber, S.H.;Souza, E. eFuriatti, R. S. 2010. Bioatividade de óleos essenciais de sassafrás e eucalipto em cascudinho. Cienc Rural, 40: 637-643.

Salin, C.; Delettre, Y.R.; Cannavaccioulo, M. and Vernon, P. 2000. Spatial distribution of Alphitobius diaperinus (Panzer) (Coleopetera: Tenebrionidae) in the soil of a poultry house along a breeding cycle. Eur J Soil Biol, 36: 107-115.

Santos, J.C.; Alves, L.F.A.; Opazo, M.A.U.; Mertz, N.R.; Marcomini, A.M.; Oliveira, D.G.P. e Bonini, A.K. 2009. Eficiência da aplicação de inseticida químico no solo para o controle de Alphitobius diaperinus (Panzer) (Coleoptera: Tenebrionidae) em aviário de frango de corte. Arq Inst Biol, 76: 417-425.

Santoro, P.H.; Neves, P.M.O.J.; Cavaguchi, S.A.; Constanski, K.; Amaro, J.T.; Alves, L.F.A. e Gomes, B.B. 2008. Controle associado de Alphitobius diaperinuse efeito de microrganismos eficazes no desenvolvimento de Beauveria bassiana. Pesq Agropec Bras, 43: 1-8.

Silva, A.A. 2005. Potencialidade da recuperação de pastagem de Brachiaria decumbens fertilizadas com cama de aviário e fontes minerais. Uberlândia (Disertação de Mestrado-ProduçãoAnimal). Programa dePós-graduação em Ciências Veterinárias. Universidade Federal de Uberlândia. 125 pp.

Steelman, D. 1996. Beetles threaten profit by damaging house insulation, carrying diseases and reducing growth, fees efficiency. Poultry Digest, 22-23.

Uemura, D.H.;Alves, L.F.A.; Opazo, M.A.U.; Alexandre, T.M.; Oliveira, D.G.P. eVentura, M.V. 2008. Distribuição e dinâmica populacional do cascudinho Alphitobius diaperinus (Coleoptera: Tenebrionidae) em aviários de frango de corte. Arq Inst Biol, 75: 429-435.

Vittori, J.; Schocken-lturrino, R. P.; Trovó, K. P.; Ribeiro, C. A. M.; Barbosa, G. G.; Souza, L.M. e Pigatto, C.P. 2007. Alphitobius diaperinus como veiculador de Clostridium perfringens em granjas avícolas do interior paulista - Brasil. Cienc. Rural, 37: 894-896.

Wolf, J.; Gouvea, A., Silva, E.R.L., Potrich, M. e Appel, A. 2014. Métodos físicos e cal hidratada para manejo do cascudinho dos aviários. Cienc Rural, 44: 161-166. 